\title{
14 CONSUMER PRIVACY AND ONLINE MARKETING: BRINGING THE HUMAN BACK INTO THE PICTURE
}

\author{
Laurence Brooks \\ Alexis Airey \\ Department of Information Systems and Computing \\ Brunel University \\ Uxbridge, Middlesex UB8 $3 P H$ \\ United Kingdom
}

\begin{abstract}
This paper investigates the possible technological solutions to consumer concerns over privacy and online marketing. It is not about how consumers can "protect" themselves from online marketers; rather, it investigates how the apparent conflict between consumers and marketers can be resolved. Based around an online survey, it finds that technologies that facilitate human interaction in e-commerce environments might provide a solution. Technologies such as chat facilities, online telephone calls, and simulated sales assistants offer an opportunity to re-establish trust. They provide all of the data gathering capabilities of traditional web technologies but with enough anonymity to allay consumers' fears. They can bring $a$ sense of the "real" world to the "virtual" world and represent the "remembering" of a consumer's details as a less alien concept.
\end{abstract}

\section{INTRODUCTION AND SURVEY DESIGN}

The aim of this study was to investigate the possible solutions to consumer concerns over privacy and online marketing, using an online survey. This aims to answer a number of questions about topics including information gathering Internet technologies (e.g., cookies in banners and e-mails), consumer pre- 
paredness to take preventative measures against information gathering technologies, the scope of anonymity and privacy policies, and consumers' receptiveness to technology that facilitates human interaction.

The sample size for this survey was over 1,000, larger than many previous surveys (e.g., Kehoe et al. 1998). Due to time restrictions, only a set of 400 responses was analyzed. However, the distribution of results from these 400 responses were not significantly different from interim results calculated at 100 and 200 responses.

The online survey was developed and pre-tested with the aid of a combination of technical and non-technical colleagues. Questions were tested for potential ambiguities and technical jargon, and the survey's code was tested for technical errors. The design of the survey was such that the respondents completed it as an online HTML form and the results were e-mailed via a CGI form. This survey is particularly useful as an indication of the evolution of the web. It was conducted specifically to examine online privacy concerns, not just to establish that they are concerned but how they are concerned. Its aim was to inform policy decisions and the development of technology tools that can assist users in protecting their privacy.

\section{ANALYSIS, EVALUATION AND CONCLUSIONS}

In order to investigate the possible technological solutions to consumer concerns over privacy and online marketing, a number of questions were asked, and the following attempts to provide insight.

\subsection{Do the Current Internet Technologies That Enable the Gathering of Information, Such as Cookies in Banners and E-mails, Have a Future?}

The future of targeted marketing through e-mail appears promising, according to the results of this survey. If a web site has a privacy policy, $76 \%$ of users will provide their e-mail address. Of respondents who receive e-mails from web sites that they have visited, $84 \%$ read them and e-mail is considered the second most popular technology to improve the buying experience. Users want to receive e-mail from sites they are interested in and will read them.

However, e-mail is severely affected by the legacy of unsolicited e-mail or "spam." The number of people who have received unsolicited e-mail remains high at $98 \%$. This high amount of junk mail is hampering the success of this medium and provoking an adverse reaction from users. Users are also taking 
actions against legitimate marketers as a consequence. Only $26 \%$ of people normally opt into e-mail mailing lists. When these people were asked their reasons, most answered that they were interested in hearing about product information but were afraid of receiving spam from other companies. With more people having access to e-mail than the Internet, e-mail has the potential to be extremely successful. However, action needs to be taken to make e-mail more acceptable in the consumer's eyes. Web sites need to distance themselves from spammers and ensure that consumers trust they will receive only the information they express an interest in.

The future of banners does not appear to be as positive. Until recently, marketers have considered banners a worthwhile investment. Of the $£ 3.1$ billion spent on online advertising last year, $56 \%$ was on banners (Mazur 2000). However, about $35 \%$ of potential online advertisers are becoming discouraged by low click through rates (Mazur 2000). The results from this survey specifically relating to banner usage do not advance the argument either way. The number of users who have, or have not, clicked on banners is fairly evenly distributed.

Until recently individual web sites have been able to match the personal data a user has entered when completing an online registration form to their browsing on that site but the user details that appear to advertising servers has been anonymous. However, the recent merger of DoubleClick with Abacus Direct Corporation established an online database consisting of personally identifiable information. DoubleClick can associate the non-personally associated information collected from a banner with personally identifiable information if the user has agreed to receive personally-tailored advertisements. Currently the user is given the opportunity when registering with an Abacus Alliance site to choose whether they want to be involved with DoubleClick.

The key point is that the element of choice open to the consumer regarding DoubleClick's tracking is the result of public opinion and a court ruling and not the original decision of DoubleClick. Indeed, public opinion appears to be the key factor affecting banners. Only $18 \%$ of users would like banners to be personalized to their interests, fewer than those preferring it when a web site is personalized. This survey found that the percentage of people that would accept a personal identifier (not identified as a cookie) decreased from $78 \%$ to $60 \%$ when it was explained that it would be used to provide personalized advertising, and then to $44 \%$ when it was explained it would be used for tracking (Cranor 1999). The method of obtaining data associated with banners can be identified as a crucial factor. Overall, the results indicate that most users do not agree that their actions should be tracked for personalization purposes or made available to marketers. 


\subsection{Are Consumers Really Prepared to Take Preventative Measures Against Technologies Such as Cookies?}

From other surveys, it appears that at a significant number of users have, at some point, tried to take action with their browsers to combat cookies. Indeed, a far higher level has been identified than accounted for previously. The low levels of action reported in previous surveys (by only asking about current browser settings) have disguised the true actions of users. CyberDialogue (Mabley 2000) reported recently that their surveys show that the number of users who accept all cookies has risen from $19 \%$ in 1997 to $46 \%$ in 1999 . Further, in this survey, we show an increase to $56 \%$ of the number of people accepting cookies. This does not, however, indicate an increasing desire to accept cookies. It is possible that as more sites adopt cookies, for example in "shopping carts," these sites will not function without cookies enabled. This survey found that $77 \%$ of people who have taken some sort of filtering action against cookies have then had to revert back to accepting cookies at some point. Even more significant is the potential for action. This survey clearly indicates that of the users who have not already used anonymizing software, $83 \%$ would consider using it.

There is a strong discrepancy between what users currently do and the action they potentially will take. That they have not taken this action can be identified as lack of knowledge of anonymizing software and the current inconvenience of using such services. Currently, anonymizing services require users to allow extra time and effort (and possibly expense), in the same way that taking browser actions against cookies does. This inconvenience is clearly identified in the survey as a factor preventing user's actions against marketing. However, anonymizing services and browser filtering are now becoming more advanced and easier to use.

\subsection{What Is the Scope of Anonymity to Allow Current Techniques to Continue and Develop?}

The survey's latter section suggests that a higher degree of anonymity on the web will become common, if current consumer opinions are a guide and developments in browser software and anonymizing services continue. To suggest to a marketer that anonymity on the web could be a solution to the conflict between consumer and marketer sounds strange. The results of the survey indicate that anonymity is a threat to marketers as opposed to a long term solution. However, Hoffman et al. (1997) identify anonymity as a temporary solution. Pseudonym services, such as those offered by Proxymate, are not a significant threat to marketers. It is still possible for companies to target effectively and reach the consumer as the e-mails are forwarded and read. 
However, this is not an ideal situation for marketers. If anonymity becomes reality, the acquisition of personally identifiable information would no longer be a possibility. Tracking would effectively be stopped also. Further developments in favor of marketers could be relied upon as a solution and an undermining factor affecting these services. In the past, anonymizing services have suffered adverse publicity as marketers have found ways around their effectiveness. There have been several revelations of the inability of these services to stop JavaScript from eliciting details from customers. However, a more effective solution would be to elicit trust from consumers so that they do not feel that they need anonymity.

\subsection{What Is the Scope of Privacy Policies to Allow Current Techniques to Continue and Develop?}

One possible solution to addressing the conflict between consumers and marketers is the implementation of privacy statements on web sites. The results of this survey show that privacy statements are a necessity, but not necessarily a solution on their own. The survey highlights the continuing confusion with regard to the capabilities and uses of cookies. There is a strong need to educate users about the uses and abilities of cookies and the privacy statement has a major role in fulfilling this. If users know exactly what information will be gathered on them, what it will be used for, and to whom it will be passed, the survey indicates that more people would volunteer information.

It is probable that users only read the privacy policies on sites where they do not recognize or trust the brand. This survey reveals that the existence of a privacy statement does not deter the giving of false details to a site, if people are inclined to do so. A possible reason for the lack of total effectiveness of privacy policies is that users do not trust companies to abide by them. The number of government bills under development aimed at instigating and policing privacy statements supports this belief.

If privacy statements are to be successful, consumers need to have faith in them. Legal approaches are a possible solution. Indeed, the majority of respondents want to see stronger laws affecting privacy. However, these laws are not a solution on their own; it is not possible to instantly instil faith.

\subsection{Are Consumers' Receptive to Technology That Will Facilitate Human Interaction?}

Jupiter reported that $90 \%$ of online shoppers prefer human interaction before making a purchase (Privacy Times 1999). This survey examines the techno- 
logies that facilitate individual human interaction and assesses acceptability relative to traditional technologies. No great strength of feeling was shown toward any of the options, either human interaction or other. Although it would have been more helpful if responses had shown swing in either direction, it is possible to speculate that users are receptive to human interaction technology. The results suggest that technologies that allow human interaction can fill the gap left by privacy policies. That industry believes this is evidenced by its adoption into web sites, particularly in the banking industry, where relationship and trust are essential.

Human interaction technologies can solve two of the major problems associated with traditional online marketing technologies. For example, users are more comfortable divulging information in real world situations than virtual ones. Human interaction technologies can also facilitate a degree of anonymity. These technologies do capture some information on users but it could be argued that they bring a desirable level of anonymity to the consumer marketer relationship, acceptable on both sides.

Human interaction technologies, although predicted as necessary in the past, have been hampered by technological developments. In particular, bandwidth and modem speeds have been a major problem. Online phone calls are becoming popular, and are being adopted by consumers for the cost savings they provide, as well as the human touch they bring. Picking up a phone is a natural reaction when users want advice and such services are expected to flourish. Often web sites cannot afford either the time or money to provide 24 hour human online services and simulated sales assistants offer a viable alternative. They are a method of providing a relationship and of accurately analyzing data. Given the high numbers of sites that have now adopted them, industry believes they will be a success.

\section{CONCLUSIONS}

The results of this privacy and marketing survey combined with current social, technological, and political events leads this paper to posit that technologies that facilitate human interaction in e-commerce environments are a potential solution to consumer concerns over privacy and online marketing.

The paper examined the future of current Internet technologies that facilitate the gathering of information. It found that the future of banners and tracking is dubious. Not only do consumers consider their presence a nuisance, but find their methods of data gathering an intrusion into their privacy. The future of email technology for marketing appears much brighter but it requires action to be taken to re-establish consumer trust that this medium will not be abused. 
The survey establishes that online users have, and are prepared, to take action to protect their privacy online. Such actions can have a negative impact, either intentionally or inadvertently, on the current popular marketing technologies. The possible solutions considered to establish a compromise between marketers and consumers are anonymity on the web, privacy statements, and human interaction technologies. The survey reveals that anonymity can be considered a temporary solution to allay consumers' concerns over privacy and online marketing. However, it does little to aid the cause of the marketer. Privacy statements are regarded as a positive step toward bridging the gap between marketer and consumer, but they must be trusted and enforced, they appear to be sufficiently neither at the moment. Technologies that facilitate human interaction offer a pathway to re-establish trust. They provide all of the data gathering capabilities of traditional web technologies but with enough anonymity to calm consumers' fears. They can bring a sense of the "real" world to the "virtual" world and represent the "remembering" of a consumer's details as a less alien concept. Used in conjunction with privacy statements, they can be doubly effective and, importantly, the survey establishes that consumers are receptive to their use.

\section{REFERENCES}

Cranor, L., Reagle, J., and Ackerman, M. Beyond Concern: Understanding Net Users'Attitudes about Online Privacy, AT\&T Labs-Research Technical Report TR 99.4.3, April 14, 1999.

Hoffman, D., Novak, T., and Peralta, M. "Information Privacy in the Marketspace: Implications for the Commercial Uses of Anonymity on the Web," November 9, 1997 (http://ecommerce.vanderbilt.edu/papers/anonymity/anonymity2_nov10.htm).

Kehoe, C., Pitkow, J., and Rogers, J. GVU's Ninth WWW User Survey Report, Atlanta: Georgia Tech Research Corporation, July 1998.

Mabley, K. “Privacy vs. Personalization: Part III," White Paper, Cyber Dialogue Inc., New York, 2000.

Mazur, L. "What Next for the Banner Ad?" Internet Business, July 2000, p. 78.

Privacy Times. "Industry Privacy Failures Hurting E-Commerce, Latest Surveys Show," Privacy Times, September 9, 1999.

\section{About the Authors}

Laurence Brooks is a lecturer in the Department of Information Systems and Computing at Brunel University. Laurence earned his doctorate at the University of Liverpool and has worked as a Research Associate at the Judge Institute of Management Studies, University of Cambridge, and as a lecturer in the Department of Computer Science, University of York. He is a board member of the UK Academy for Information Systems (UKAIS). He is a member of the 
IFIP and UK Northern Interest Group (NISG) for IS and organizations. He was the publicity chairman for the IEEE Symposium on Requirements Engineering (RE '95) held in York in March 1995 and co-chaired the UKAIS annual conference in 1999. He has published in journals such as the European Journal of Information Systems, Information Systems Journal, and Information and Software Technology. He can be reached by e-mail at laurence.brooks@ brunel.ac.uk.

Alexis Airey gained a Master's in Information Processing from the Department of Computer Science at the University of York. She is working as a Mediation Systems Delivery Manager for Information Systems Engineering in British Telecom. Her e-mail address is alexis.airey@bt.com. 